\title{
Simulation of resonant tunneling heterostructures: numerical comparison of a complete Schrödinger-Poisson system and a reduced nonlinear model
}

\author{
Virginie Bonnaillie-Noë* \\ IRMAR, UMR CNRS 6625, ENS Cachan Bretagne, av Robert Schuman, F-35170 Bruz, FRANCE.
}

Ali Fara

MIP, UMR CNRS 5640, Université Paul Sabatier, F-31062 Toulouse Cedex 9, FRANCE

Francis Nie1

IRMAR, UMR CNRS 6625, Université de Rennes I, F-35042 Rennes Cedex, FRANCE

(Dated: March 27, 2008; Received March 27, 2008)

\begin{abstract}
Two different models are compared for the simulation of the transverse electronic transport through an heterostructure: a $1 D$ self-consistent Schrödinger-Poisson model with a numerically heavy treatment of resonant states and a reduced model derived from an accurate asymptotic nonlinear analysis. After checking the agreement at the qualitative and quantitative level on quite well understood bifurcation diagrams, the reduced model is used to tune double well configurations for which nonlinearly interacting resonant states actually occur in the complete self-consistent model.
\end{abstract}

\section{INTRODUCTION}

The modelling of the electronic transport in quantum electronic devices is well described within a Landauer-Büttiker approach (see [1][2][3][4][5][6][7]). A well-known difficulty for the numerical simulations comes from quantum resonances because they produce very stiff variations of the spectral quantities with respect to the energy variable: this increases dramatically the numerical complexity or requires some specific treatment. This point becomes an issue when the numerical simulation is motivated by a self-consistent nonlinear problem which takes into account in a mean field approach the electronic repulsion (see [8][9] 10]). Such a nonlinear system is often referred to as a Schrödinger-Poisson system. Meanwhile in the one-dimensional setting the scattering states are simply described in the active region by using energy-dependent transparent boundary conditions for the Schrödinger equation. After an accurate asymptotic analysis of the nonlinear spectral problem presented in [11] 12] [13], an asymptotic reduced model has been derived by considering the regime of a finite number of resonant states produced by quantum wells in a semiclassical island. The reduced model summarizes how a finite number of resonant states as well as the phase-space geometry of the tunnel effect governs the nonlinearity and may produce several nonlinear solutions like in [14]. After introducing carefully the scaling and checking the relevancy of the approximations, realistic cases similar to the one studied in [10] for $\mathrm{GaAs}$ devices and [9] for $\mathrm{Si}_{-} \mathrm{SiO}_{2}$ devices have been numerically tested in [15]. The agreement with the simulations of [10][9] were satisfactory although the configurations in [15] were not exactly the same (for example, the nonlinear effect outside the barriers were not taken

\footnotetext{
*Electronic address: Virginie.Bonnaillie@ bretagne.ens-cachan.fr

†Electronic address: faraj@mip.ups-tlse.fr

‡Electronic address: Francis.Nier@univ-rennes1.fr
}

into account although they are known to produce sometimes non negligible effects, see for example [16]). Furthermore the rapidity of the computations and the exhaustive description of nonlinear solutions with the finite dimensional reduced model made possible the exploration of some exotic nonlinear solutions with nonlinearly interacting resonant states. The purpose of the present article is twofold

1. Check by taking exactly the same data for both models that the reduced model and the complete SchrödingerPoisson model agree very well at the qualitative and quantitative level.

2. Check that the exotic nonlinear solutions found in the double well problem make sense within the Schrödinger-Poisson problem and lead to interesting physical phenomena like a damped beating effect stable for an extended range of applied bias.

Although a rescaling leading to dimensionless quantities allows to consider equally $\mathrm{Ga}-\mathrm{As}$ or $\mathrm{Si}-\mathrm{SiO}_{2}$ devices (see [15]) only $\mathrm{Ga}$-As have been considered here.

\section{THE MODEL}

\section{Initial writing.}

The mass $m$ that we use is the effective electronic mass $m=$ $m_{3}$ in the transverse direction $\underline{x}=\underline{x}_{3}$. The quantum hamiltonian for a single electron has the form

$$
-\frac{\hbar^{2}}{2 m} \frac{d^{2}}{d \underline{x}^{2}}+\underline{\mathcal{V}}(\underline{x}), \quad \underline{\mathcal{V}}=\underline{\mathcal{B}}+\underline{\mathcal{V}}_{0}+\underline{V}_{N L},
$$

with a nonlinear potential $\underline{V}_{N L}$ which is non negative and takes into account the mean repulsive electrostatic potential in the area $a \leq x \leq b$. The nonlinear effects are not taken into account outside the heterostructure (quasineutral approximation). The total potential denoted by $\underline{V}$ also includes the 
piecewise affine function $\underline{\mathcal{B}}$ referring to the bias voltage $-\underline{B}$,

$$
\underline{\mathcal{B}}(\underline{x})=-\underline{B}\left[\frac{\underline{x}-a}{b-a} 1_{[a, b]}(\underline{x})+1_{[b,+\infty)}(\underline{x})\right] .
$$

It is also made of the second term $\mathcal{V}_{0}$ which describes the barriers and the wells,

$$
\underline{\mathcal{V}}_{0}(\underline{x})=\underline{V}_{0} 1_{[a, b]}(\underline{x})+\sum_{j=1}^{N} \underline{W}_{j}(\underline{x}),
$$

with the constant $\underline{V}_{0}>0$ and the compactly supported potentials $\underline{W}_{j} \in L^{\infty}(\mathbb{R}),-\underline{V}_{0} \leq \underline{W}_{j} \leq 0$, fixed. The external potential $\underline{\mathcal{B}}+\underline{\mathcal{V}}_{0}$ is represented in Figure 1 The beam of electrons

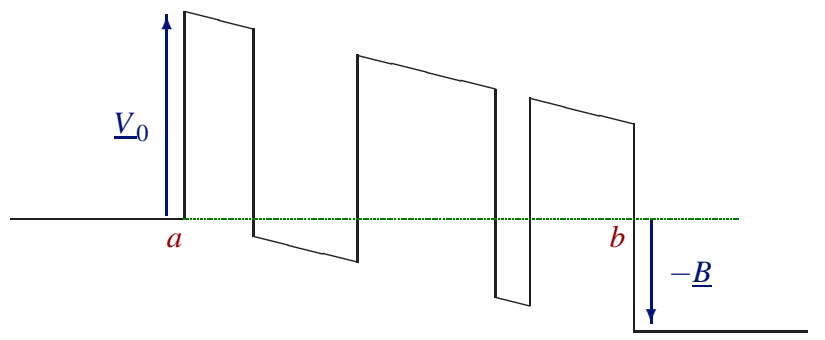

FIG. 1: Linear potential in the heterostructure.

injected from both sides is described by a function $\underline{f}$ of the momentum variable $k \gtrless 0$

$$
\underline{f}(\underline{k})=\underline{g}_{+}\left(\underline{k}^{2}\right) 1_{\mathbb{R}_{+}}(\underline{k})+\underline{g}_{-}\left(\underline{k}^{2}-B\right) 1_{\mathbb{R}_{-}}(\underline{k}) .
$$

Up to the reference energy $(0$ or $-\underline{B})$, the injection profile is the same for both sides

$$
\underline{g}_{+}(E)=\underline{g}_{0}(E) \quad \text { and } \quad \underline{g}_{-}(E)=\underline{g}_{0}(E+B),
$$

where $g_{0}$ is the Fermi-Dirac distribution function integrated along the two directions $\left(\underline{x}_{1}, \underline{x}_{2}\right)$

$$
\underline{g}_{0}\left(\underline{k}^{2}\right)=\frac{\sqrt{m_{1} m_{2}} k_{B} T}{\pi \hbar^{2}} \ln \left(1+\exp \left(\frac{E_{F}-\frac{\hbar^{2} k^{2}}{2 m}}{k_{B} T}\right)\right),
$$

with the Fermi level

$$
E_{F}=\frac{\hbar^{2}}{2}\left(3 \pi^{2} n_{D}\right)^{2 / 3}\left(m_{1} m_{2} m_{3}\right)^{-1 / 3} .
$$

The introduction of a non isotropic effective electronic mass $\left(m_{1}, m_{2}, m_{3}\right)$ with this simplified relation between donor density $n_{D}$ and $E_{F}$ allows to adapt this model and the numerical simulations to $\mathrm{Si}_{-} \mathrm{SiO}_{2}$ heterostructures like in [9].

The out-of-equilibrium regime for Schrödinger-Poisson system requires the introduction of the generalized eigenfunctions in order to describe the steady state density. The incoming generalized eigenfunctions $\underline{\psi}_{-}(\underline{k}, \underline{x})$ are defined for $\underline{k}>0$ by

$$
\begin{aligned}
-\frac{\hbar^{2}}{2 m} \frac{d^{2}}{d \underline{x}^{2}} \underline{\Psi}_{-}(\underline{k}, \underline{x})+\underline{\mathcal{V}}_{\underline{\Psi}} \underline{\Psi}_{-}(\underline{k}, \underline{x})=\frac{\hbar^{2} \underline{k}^{2}}{2 m} \underline{\Psi}-(\underline{k}, \underline{x}) & \text { for } \underline{x} \in \mathbb{R}, \\
\underline{\Psi}_{-}(\underline{k}, \underline{x})=\mathrm{e}^{i \underline{k} \underline{x}}+R(\underline{k}) \mathrm{e}^{-i \underline{k} \underline{x}} & \text { for } \underline{x} \leq a, \\
\underline{\Psi}_{-}(\underline{k}, \underline{x})=T(\underline{k}) \mathrm{e}^{i \sqrt{\underline{k}^{2}+\underline{B}} \underline{x}} & \text { for } \underline{x} \geq b,
\end{aligned}
$$

with a similar formulation for $\underline{k}<0$ (exchange $x \leq a$ and $x \geq b$, replace $\underline{k}$ by a well chosen square root of $\underline{k}^{2}-\underline{B}$, see [15] for details). The electronic density $\underline{n}$ is given by

$$
\begin{aligned}
\underline{n}(\underline{x})=\int_{0}^{+\infty} \underline{g}\left(\underline{k}^{2}\right) \mid \underline{\Psi}_{-} & \left.(\underline{k}, \underline{x})\right|^{2} \frac{d \underline{k}}{2 \pi} \\
& +\int_{-\infty}^{0} \underline{g}\left(\underline{k}^{2}-B\right)\left|\underline{\Psi}_{-}(\underline{k}, \underline{x})\right|^{2} \frac{d \underline{k}}{2 \pi} .
\end{aligned}
$$

Finally the nonlinear potential $\underline{V}_{N L}$ solves the Poisson equation

$$
\left\{\begin{array}{l}
-\Delta \underline{V}_{N L}=\frac{q^{2}}{\varepsilon} \underline{n}, \\
\underline{V}_{N L}(a)=\underline{V}_{N L}(b)=0 .
\end{array}\right.
$$

The average current density (independent of $\underline{x}$ in the steady state) is computed according to the steady state formula

$$
\underline{J}=\frac{e \hbar}{m} \int_{0}^{+\infty} \frac{g\left(\underline{k}^{2}\right)}{b-a} \int_{a}^{b} \mathfrak{I} \nabla \underline{\psi} \underline{\underline{h}}(\underline{k}, \underline{x}) \underline{\underline{\psi} \underline{h}(\underline{k}, \underline{x})} d \underline{x} \frac{d \underline{k}}{2 \pi} .
$$

\section{Rescaling.}

For a more flexible numerical treatment which can be adapted to several kinds of semiconductors, and also in order to carry out the asymptotic analysis which has led to the reduced model, the Schrödinger-Poisson system is better written with dimensionless quantities and unknowns. The rescaled position variable $x=(\underline{x}-a) / L, L=b-a$, now lies in $[0,1]$ and the rescaled energies are given by $E=\underline{E} / E_{F}, V=\underline{V} / E_{F}$. The nonlinear system with unknow $V_{N L}^{h}$ can be written

$$
\left\{\begin{array}{l}
-h^{2} \frac{d^{2}}{d x^{2}} \psi_{-}^{h}(k, x)+\mathcal{V}^{h} \Psi_{-}^{h}(k, x)=k^{2} \psi_{-}^{h}(k, x), \\
\quad+\text { transparent boundary conditions at } x=0 \text { or } 1, \\
\mathcal{V}^{h}(x)=\mathcal{B}(x)+\mathcal{V}_{0}^{h}(x)+V_{N L}^{h}(x), \\
\mathcal{V}_{0}^{h}(x)=V_{0} 1_{[0,1]}(x)-\sum_{j=1}^{N} W_{j}\left(\frac{x-c_{j}}{h}\right), \\
g_{0}\left(k^{2}\right)=\beta^{-1} \ln \left(1+\exp \left(\beta\left(1-k^{2}\right)\right)\right) \\
g_{+}(E)=g_{0}(E) \quad \text { and } \quad g_{-}(E)=g_{0}(E+B), \\
n(x)=\int_{0}^{+\infty} g_{+}\left(k^{2}\right)\left|\psi_{-}^{h}(k, x)\right|^{2} \frac{d k}{2 \pi h} \\
\quad+\int_{-\infty}^{0} g_{-}\left(k^{2}-B\right)\left|\psi_{-}^{h}(k, x)\right|^{2} \frac{d k}{2 \pi h} \\
-\Delta V_{N L}^{h}=\gamma n, \quad \text { with } \quad V_{N L}^{h}(0)=V_{N L}^{h}(1)=0 .
\end{array}\right.
$$

The parameters equal

$k=\frac{\underline{k} \hbar}{\sqrt{2 m E_{F}}}, \quad h=\frac{\hbar}{L \sqrt{2 m E_{F}}}, \quad \beta=\frac{E_{F}}{k_{B} T} \quad$ and $\quad \gamma=\frac{4 L}{a_{B}}$, where the Bohr radius is defined as usual $a_{B}=\frac{4 \pi \hbar^{2} \varepsilon}{\sqrt{m_{1} m_{2}} q^{2}}$.

\section{THEORY AND APPLICATIONS}

The reduced model is obtained after taking the limit $h \rightarrow 0$ in the system (6). This analysis has been carried out 
completely in the series of articles $[12][13][11]$. In the end the reduced model consists in solving some simple collection of finite dimensional (the dimension equals the number $N$ of wells) nonlinear systems with constraints. In order to write them, some notations are necessary. We simply give a brief review of the theoretical results and their application. The adaptation of this system in realistic situations when $h>0$ is 0.1 or 0.3 has been explained with details in [15].

\section{Notations.}

- For a given limit $V=\lim _{h \rightarrow 0} V_{N L}^{h}$, the potential $\tilde{\mathcal{V}}$ is the corresponding filled (i.e. where the wells $W_{j}$ have been removed) potential

$$
\tilde{\mathcal{V}}(x)=\mathcal{B}(x)+V_{0} 1_{[0,1]}(x)+V(x) .
$$

- For any $j=1, \ldots, N,\left(-\varepsilon_{j}^{k}\right)_{1 \leq k \leq K_{j}<+\infty}$ denotes the finite collection of negative eigenvalues for the Schrödinger operator $H_{j}:=-d^{2} / d x^{2}+W_{j}(x)$, labelled in the increasing order. The set of energies $\mathcal{E}_{j}$ is defined by

$$
\mathcal{E}_{j}=\left\{\tilde{\mathcal{V}}\left(c_{j}\right)-\varepsilon_{j}^{k}, 1 \leq k \leq K_{j}\right\} .
$$

- The set of resonant energies is defined as $\mathcal{E}=\cup_{j=1}^{N} \mathcal{E}_{j}$.

- For any $E \in \mathbb{R}$, we set

$$
J^{E}:=\left\{j \in\{1, \ldots, N\} \quad \text { s.t. } \quad E \in \mathcal{E}_{j}\right\} .
$$

When $j \in J^{E}$, the well $c_{j}$ is called resonant at the energy E.

- Finally, we set

$$
c_{\ell}^{E}:=\min _{j \in J^{E}} c_{j}, \quad c_{r}^{E}:=\max _{j \in J^{E}} c_{j},
$$

and simply

$$
c^{E} \quad \text { when } \quad c_{\ell}^{E}=c_{r}^{E} .
$$

- For a potential $\Phi$, the action (or Agmon distance) is given by

$$
d_{A g}(x, y ; \Phi)=\left|\int_{x}^{y} \sqrt{\max \{\Phi(t), 0\}} d t\right| .
$$

For a resonant energy $E \in \mathcal{E}$, we set

$$
\begin{aligned}
& \delta_{\ell}^{E}:=d_{A g}\left(c_{r}^{E}, 1 ; \tilde{\mathcal{V}}-E\right)-d_{A g}\left(0, c_{r}^{E} ; \tilde{\mathcal{V}}-E\right), \\
& \delta_{r}^{E}:=d_{A g}\left(0, c_{\ell}^{E} ; \tilde{\mathcal{V}}-E\right)-d_{A g}\left(c_{\ell}^{E}, 1 ; \tilde{\mathcal{V}}-E\right) .
\end{aligned}
$$

\section{Asymptotic system.}

The reduced model written for all the possible limits $V$ of $V_{N L}^{h}$ as $h \rightarrow 0$ is written as a simple Poisson equation

$$
\left\{\begin{array}{l}
-\Delta V=\sum_{E \in \mathcal{E}} \sum_{j \in J^{E}}\left(t_{j}^{E}\left(g_{+}(E)-g_{-}(E)\right)+g_{-}(E)\right) \delta_{c_{j}}, \\
V(0)=V(1)=0
\end{array}\right.
$$

with the convention that $\left(g_{+}-g_{-}\right)(0)$ can be any value in $\left[0,\left(g_{+}-g_{-}\right)\left(0^{+}\right)\right]$and where the coefficients $t_{j}^{E}$ belong to $[0,1]$ and satisfy:
- $t_{j}^{E}=0$ when $E<0$,

- and for $E>0$,

$$
\left\{\begin{array}{l}
\delta_{\ell}^{E}>0 \Rightarrow t_{j}^{E}=1, \forall j \in J^{E} \\
\delta_{r}^{E}>0 \Rightarrow t_{j}^{E}=0, \forall j \in J^{E}
\end{array}\right.
$$

The above system (8) is actually a finite dimensional system since the charges are asymptotically concentrated like deltafunctions and the potential $V$ is a piecewise affine function. This asymptotic system restates the paradigm about resonant heterostructures which says that the nonlinear effects are governed by a finite number of resonant states. The quantum mechanics is contained in the finite set of resonant energies and also in the coefficients $t_{j}$ which encodes the comparison of the tunnel effects between the left- and right-hand sides. Consider the single well case for example: the coefficient $t_{1}$ vanishes when the tunnel effect at the resonant energie is easier on the left-hand side than on the right-hand side, equals 1 in the opposite case, and can take an arbitrary value when the two tunnel effects have a comparable intensity. The general rule 9 given above is just an example of a possible comparison. Things can be specified and are a bit more complicated when the interaction of resonances is made possible in the multiple well problem. On the basis of the theoretical analysis carried out in [12][13], a complete classification has been proposed in [15] for the double well problem. Finally note that the coefficients $t_{j}$ and the value $\left(g_{+}-g_{-}\right)(0)$ can be viewed as Lagrange parameters which takes arbitrary value when some constraint about the Agmon distances or the energies is saturated. Hence the finite dimensional problem can be easily solved numerically in order to get all the possible asymptotic solutions to the nonlinear problem.

\section{Adaptation.}

The theoretical asymptotic analysis suggests that in the limit $h \rightarrow 0$ the quantum wells and the charges are concentrated at some points $c_{i}, i=1, \ldots, N$. In realistic cases, the rescaling of the equations leads to value of $h$ of order $10^{-1}$, which is small but not very small. The asymptotic picture has to be adapted in order to make the most suitable approximation for the different quantities: Concentrated charges is a reasonable approximation when the nonlinearity is not very strong and if $c_{i}$ is well chosen, but the comparison of the tunnel effect has to be done with the exact sizes of the potential barriers:

- The resonances have imaginary parts (or resonances width) of order $\mathrm{e}^{-C / h}$ and give rise to very stiff variations of spectral quantities even when $h>0$ is not very small. This part of the asymptotic model (i.e. the sum over the set $J^{E}$ ) is kept.

- When the nonlinearity is not very strong in comparison with the potential barrier (that is $V_{0}=\underline{V}_{0} / E_{F} \gg 1$ ), a good average position of the charge can be determined via an interpolation method based on the FeynmannHellman relation.

- The evaluation of the tunnel effects in the phasespace involves the comparison of quantities looking like 
$\mathrm{e}^{-A_{1}(h) / h}$ and $\mathrm{e}^{-A_{2}(h) / h}$. In order to make an accurate comparison of two such quantities, the factors $A_{1}(h)$ and $A_{2}(h)$ have to be computed accurately. In this case the exact geometry of the potential barriers is taken into account, that is they are computed with the exact numerical value of $h$.

Details of these modifications of the asymptotic model have been explained with details in [15] while checking at every step the relevancy of the approximations.

\section{STEADY STATES COMPUTATIONS}

In [15], a rather good numerical agreement with other simulations for $\mathrm{Ga}-\mathrm{As}$ in [10] and for $\mathrm{Si}-\mathrm{SiO}_{2}$ in [9] heterostructures was observed. The reduced model, after adaptation, allowed to understand clearly how the numerical data influence the bifurcation diagrams of nonlinear steady states when the applied bias varies. For example the hysteresis phenomenon appears clearly in a double barrier structure (one well) when the right-hand side barrier is larger than the first one and not in the opposite case. Contrary to the general numerical tests, the case when the two barriers have the same size has to be avoided since the tunnel effects on both sides are comparable, with fluctuations due to the nonlinearity and the applied bias which cannot be easily determined a priori. In the double well problem (three barriers) a possible nonlinear interaction of two resonant states, giving rise to a new branch of nonlinear steady states, was detected with the reduced model although no such case had been presented before.

The comparison with the numerical solution of the initial Schrödinger-Poisson system has two aims: 1) Verify accurately the numerical agreement between the adapted reduced model and the Schrödinger-Poisson system roughly observed in [15] when the modelling and the numerical data are exactly the same (for example the nonlinear effects outside the heterostructure are not taken into account in the present version of the reduced model contrary to the results in [10] and [9]);2) Check that the bifurcation diagrams observed in the reduced model, with a great sensitivity to numerical data, can really be produced with the far from equilibrium Schrödinger-Poisson system.

The reduced resolution is compared with the direct resolution presented in [10]. The direct resolution, considered as the reference, is performed as follows. The nonlinear system (6) is solved by using a Gummel iteration, proposed in [17], which corresponds to finding the solution $V_{N L}^{\text {new }}$ of the equation

$$
-\frac{d^{2}}{d x^{2}} V_{N L}^{\text {new }}=\gamma n^{\text {old }} \exp \left(V_{N L}^{\text {old }}-V_{N L}^{\text {new }}\right),
$$

for a given iterate $V_{N L}^{\text {old }}$. The density $n^{\text {old }}$ is computed from the potential $V_{N L}^{\text {old }}$ by solving a large enough number of Schrödinger equations, appearing in [6, in order to make an accurate numerical integration with respect to the $k$ variable in the computation of the particle density. The number of Schrödinger equations and the accuracy of the density are improved by refining the mesh of integration around the resonant energies. The convergence of the algorithm depends strongly on the initial guess, especially when several nonlinear solutions are possible. In particular, the hysteresis phenomenon can be observed numerically. Solving the system (6) by increasing and then decreasing the applied bias does not provide the same branch of solution after initializing the Gummel algorithm within a continuation method. Except with the extreme values of $B$, the numerical solution after convergence for the previous value of the bias is used as an initial guess for the Gummel algorithm. For the extreme values of $B$, the nonlinear potential is initialized to the null potential. Compared with this, the numerical resolution of the reduced model provides at once all the possible nonlinear solutions for a given bias and all the possible branches of the bifurcation diagram when the bias varies.

\section{Double barrier Ga-As heterostructure.}

In [10], Pinaud did not observe the hysteresis phenomenon because he took a symmetric double barrier. A simple change of the size of the barriers leads to multiple nonlinear solutions to the reduced model for a fixed bias, interpreted as an hysteresis case with respect to the variation of the bias. Let us recall some physical parameters:

\begin{tabular}{ll|ll} 
Rel. el. mass & 0.067 & Rel. permitivity & 11.4 \\
Temperature & $300 \mathrm{~K}$ & Height of barriers & $0.3 \mathrm{eV}$ \\
Donor density & $10^{24} \mathrm{~m}^{-3}$ & Fermi level $E_{F}$ & $0.054 \mathrm{eV}$
\end{tabular}

The numerical computations are carried out for 200 values of the applied bias $\underline{B}$ between $0 \mathrm{eV}$ and $0.25 \mathrm{eV}$ and the small parameter takes here the value

$$
h=0.22,
$$

when the geometry of the potential is given by

$$
\begin{array}{ll}
\text { Size of barriers } & 3 \times 10^{-9}, 6 \times 10^{-9} \mathrm{~m} \\
\text { Size of well } & 6 \times 10^{-9} \mathrm{~m} .
\end{array}
$$

Figure 2 gives the comparison of the I-V curves.

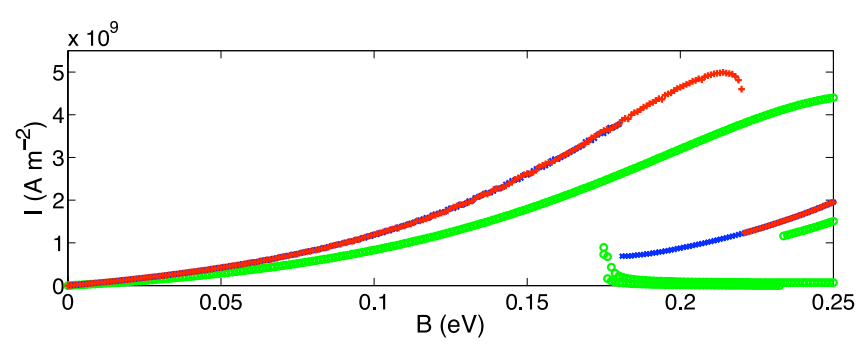

FIG. 2: Comparison of I-V curves for the two models. 
Two methods for solving the complete Schrödinger-Poisson system have been tested: 1) a continuation method by increasing or decreasing the applied bias; 2) for any fixed bias, initiate the Gummel algorithm by the series of all possible nonlinear potentials solving the reduced model. Both methods gave the same result. It is interesting to note that the hysteresis effect detected via the asymptotic model appears in the continuation method: Only one part of the I-V curve is obtained by increasing or decreasing the bias (see Fig. 3 .
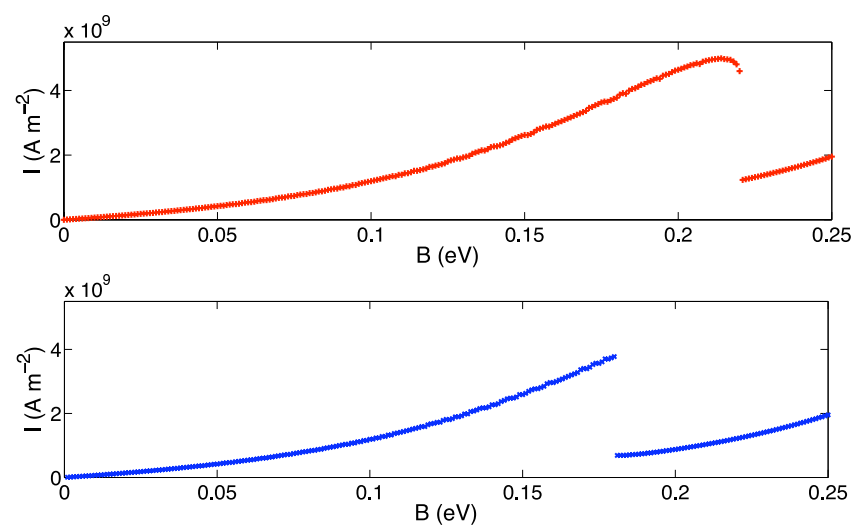

FIG. 3: Comparison of the I-V curves obtained via the continuation method by increasing (top) or decreasing (bottom) the bias.

Another way to understand or compare the bifurcation diagrams is by looking at the resonant energy attached to any nonlinear solution as a function of the applied bias. The first picture of Figure 4 shows that the bifurcation diagrams are coherent although the hysteresis phenomena are slightly amplified in the asymptotic model. The second picture of Figure 4 shows the comparison for the bias $\underline{B}=0.05 \mathrm{eV}$ of the real nonlinear potential and the piecewise affine potential of the reduced model.
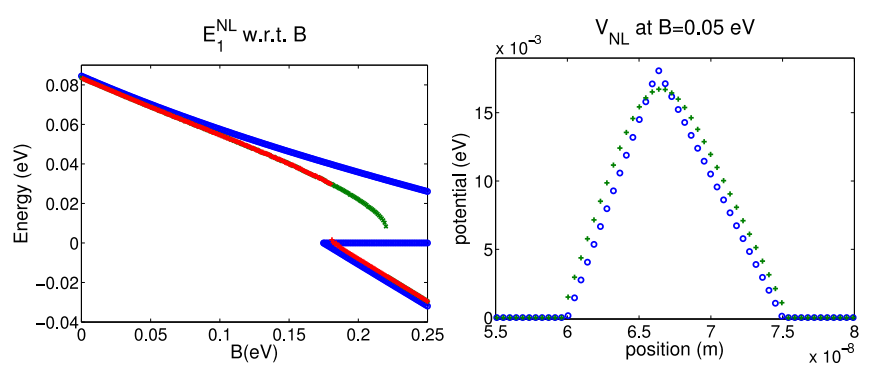

FIG. 4: Comparison of the bifurcation diagram on the $E_{\text {res }}-\mathrm{V}$ curve (left) of the nonlinear potential for $\underline{B}=0.05 \mathrm{eV}$ (right).

\section{Double well Ga-As heterostructure.}

With the same physical parameters (except the donor density and the geometry of the barriers) some cases have been tested with the reduced model with a double well (three barriers) so that there is a non trivial interaction of resonances. Such a case has already been presented in [15] for an $\mathrm{Si}_{-} \mathrm{SiO}_{2}$ heterostructure, but we can keep the same material as above with the same effect. The specific data of the device are given in Table【

$\begin{array}{ll}\text { Size of barriers } & 5 \times 10^{-9}, 5 \times 10^{-9}, 6 \times 10^{-9} \mathrm{~m} \\ \text { Size of wells } & 6 \times 10^{-9} \mathrm{~m} \\ \text { Donor density } & 5 \times 10^{24} \mathrm{~m}^{-3}\end{array}$

TABLE I: Data for the Ga-As device.

The reduced model leads to the bifurcation diagram given in Figure 5 represented in terms of the two resonant energies with respect to the applied bias (the highest resonant energy is initially in the right-hand well).

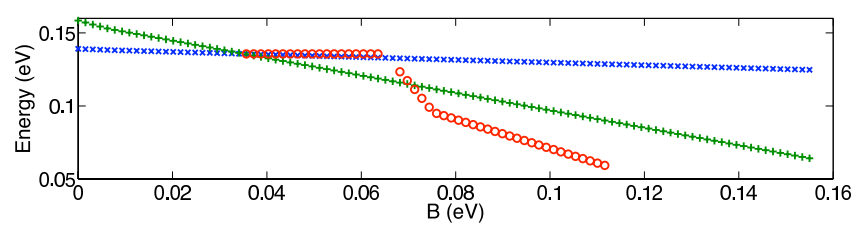

FIG. 5: Bifurcation diagram of the reduced model.

Two branches are possible when the resonances reach the same value, either they cross or they remain together due to the nonlinear effect for a wide range of the bias. Actually we know that this picture is incorrect when $h>0$ since crossings have to be replaced by avoided crossings in 1D quantum mechanical systems (although resonances can lead exceptionally to higher multiplicity Jordan blocks according to [18]). The question is to know which configuration of Figure 6 is selected by the complete Schrödinger-Poisson system.
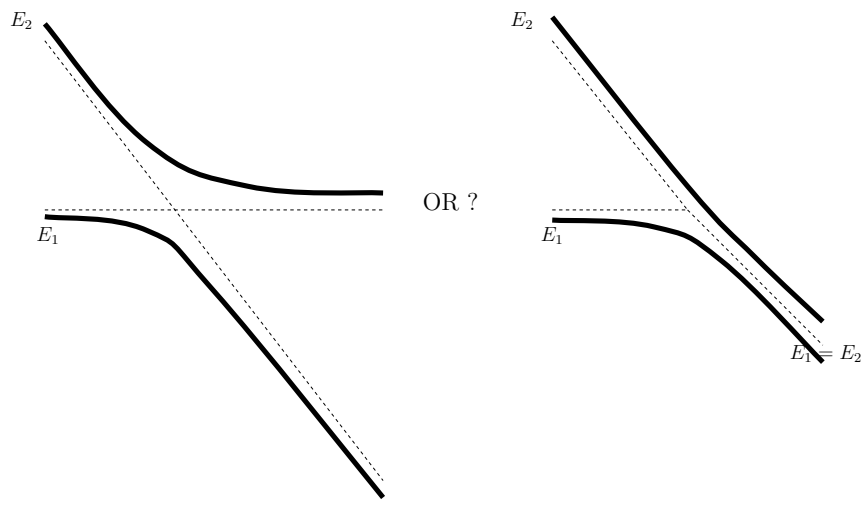

FIG. 6: Two possibilities of nonlinear avoided crossings.

The computations on the full Schrödinger-Poisson system show that both solutions coexist according to the prediction of the reduced model. 


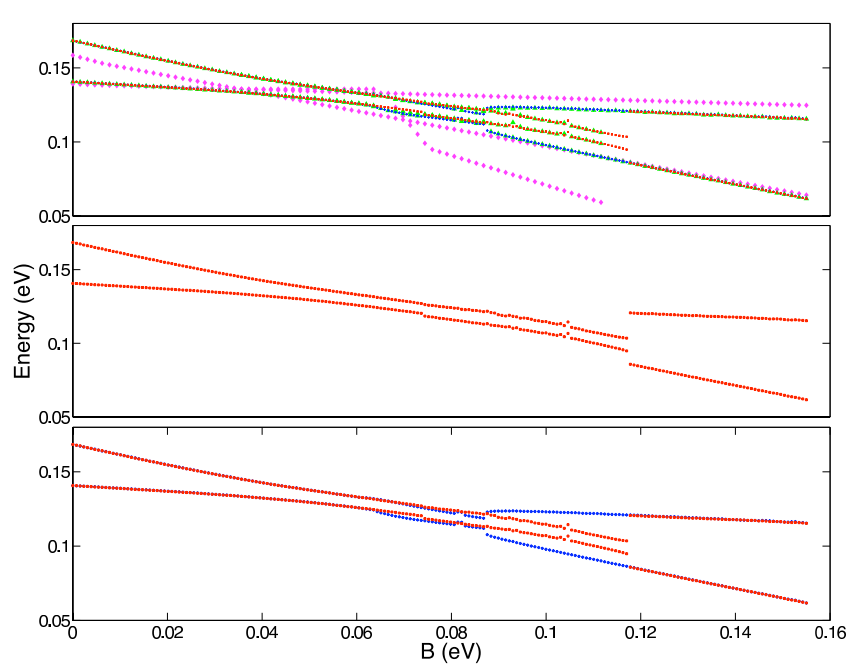

FIG. 7: Top: Comparison of the complete bifurcation diagrams; Middle: Continuation according to $\underline{B}: 0 \mathrm{eV} \rightarrow 0.16 \mathrm{eV}$; Bottom: Continuation both ways.

The I-V curves are similar (see Fig. 8.
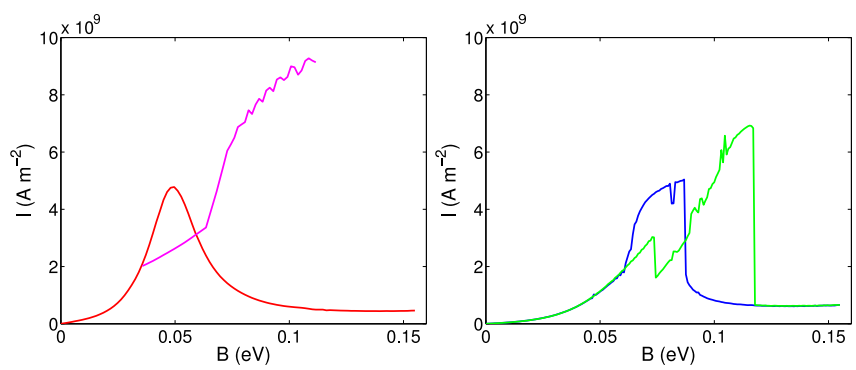

FIG. 8: Comparison of the I-V curve given by the reduced model (left) and by solving the complete Schrödinger-Poisson system (right).

The two model agree at the qualitative and quantitative level although a change by $0.5 \mathrm{~nm}$ (a few atomic layers) of the last barrier changes dramatically the bifurcation diagram of the reduced model. With its numerical efficiency, the reduced model appears here as a good tool to detect rapidly the configurations which lead to interesting nonlinear effects.

\section{TIME DEPENDENT NONLINEAR DYNAMICS}

When the barriers are well chosen, the double well problem leads to a branch of nonlinear steady states with interacting resonant states. The theoretical analysis as well as the observation of the electron density for these nonlinear solutions, show that they are due to resonant states delocalized in both wells. The question is whether this can lead to some nonlinear beating effect.
Like in [10], the time dependent Schrödinger system is solved by using a Crank-Nicholson scheme:

$$
\begin{aligned}
i \hbar \frac{\underline{\psi}^{n+1}(\underline{k}, \underline{x})-\underline{\psi}^{n}(\underline{k}, \underline{x})}{\Delta t} & =-\frac{\hbar^{2}}{2 m} \frac{d^{2}}{d \underline{x}^{2}}\left(\underline{\psi}^{n+1}(\underline{k}, \underline{x})+\underline{\psi}^{n}(\underline{k}, \underline{x})\right) \\
& +\underline{\mathcal{V}}^{n+1 / 2}(\underline{x})\left(\underline{\Psi}^{n+1}(\underline{k}, \underline{x})+\underline{\Psi}^{n}(\underline{k}, \underline{x})\right),
\end{aligned}
$$

where $\underline{\mathcal{V}}^{n+1 / 2}=2 \underline{\mathcal{V}}^{n}-\underline{\mathcal{V}}^{n-1 / 2}$ and $\Delta t$ is the time step, together with equation

$$
\left\{\begin{array}{l}
-\Delta \underline{V}_{N L}^{n+1}=\frac{q^{2}}{\varepsilon} \underline{n}^{n+1}, \\
\underline{V}_{N L}^{n+1}(a)=\underline{V}_{N L}^{n+1}(b)=0 .
\end{array}\right.
$$

The Crank-Nicholson scheme comes with the transparent boundary conditions proposed by [19] and the initial condition $\psi^{0}(\underline{k}, \underline{x})=\phi(k, x)$, where $\phi(k, x)$ denotes the wave function at frequency $k$ corresponding to the solution of 6 for the initial bias. The density $\underline{n}^{n+1}(\underline{x})$ is computed from the $\underline{\psi}^{n+1}(\underline{k}, \underline{x})$ using formula (3).

Several time-dependent simulations of the full SchrödingerPoisson system have been carried out. It is realized by switching at time $t=0$, the bias from $\underline{B}=0 \mathrm{eV}$ to $\underline{B}=0.08 \mathrm{eV}$. The initial data are the one associated with the nonlinear steady state for $\underline{B}=0 \mathrm{eV}$. Figure 9 shows the variations with respect to time of average density current calculated numerically, for the donor density $5 \times 10^{24} \mathrm{~m}^{-3}$. The average current density $\underline{J}^{n}$ at the $n$-th time step is computed by using formula (5) (such an expression makes sense within an adiabatic approximation), where the stationary wave functions are replaced by the $\underline{\psi}^{n}(\underline{k}, \underline{x})$ :

$$
\underline{J}^{n}=\frac{e \hbar}{m} \int_{0}^{+\infty} \frac{g}{\bar{b}\left(\underline{k}^{2}\right)} \int_{a}^{b} \mathfrak{I} \nabla \underline{\Psi}^{n}(\underline{k}, \underline{x}) \overline{\underline{\Psi}^{n}(\underline{k}, \underline{x})} d \underline{x} \frac{d \underline{k}}{2 \pi} .
$$
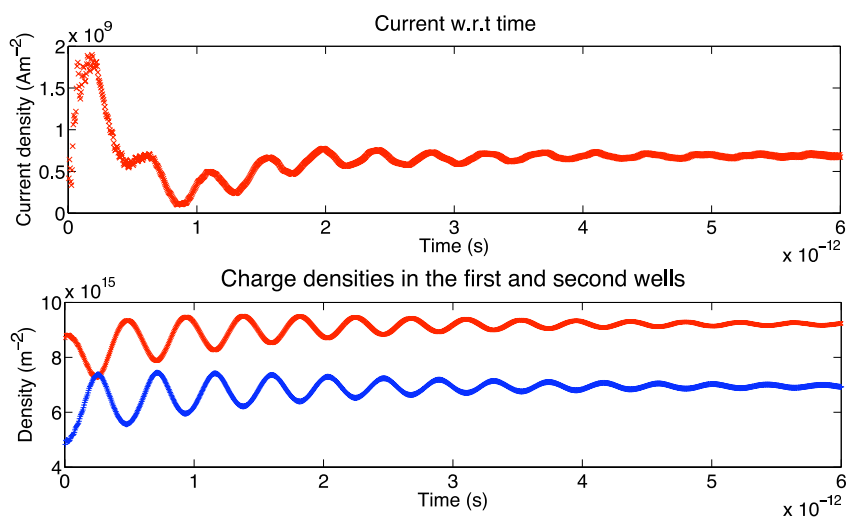

FIG. 9: Evolution of the current and the charge densities in the wells.

The (damped) beating effect is even more obvious on the second plot of Figure 9 which shows the time evolution of the charge concentrated in the first and second wells. The period of the oscillations and the damping time can be determined after applying a FFT analysis to the time dependent curves. 
The damping time is determined after translating the timedependent curve by one period $T_{p e r}$, the peak of the Fourier transform is multiplied by $\mathrm{e}^{-T_{p e r} / T_{\text {damp }}}$. The numerical computation of the period $T_{\text {per }}$ shows a good agreement with the theoretical value $2 \pi \hbar / \Delta E$, where $\Delta E$ denotes the gap (computed numerically on the nonlinear steady state solution) between the two resonant energies, although the nonlinear effects should bring some correction on the time dependent analysis. This has been done in Table $\amalg$ for several values of the doping density, without changing the other parameters. The nonlinear effects increase when the donor density is increasing.

\begin{tabular}{cccc}
\hline Donor density $\left(\mathrm{m}^{-3}\right)$ & $\frac{2 \pi \hbar}{\Delta E}(p s)$ & $T_{\text {per }}(p s)$ & $T_{\text {damp }}(p s)$ \\
\hline $10 \times 10^{24}$ & 0.69 & 0.55 & 1.1 \\
$5 \times 10^{24}$ & 0.51 & 0.43 & 1.6 \\
$2 \times 10^{24}$ & 0.54 & 0.43 & 2.1 \\
$1 \times 10^{24}$ & 0.5 & 0.43 & 2.6 \\
\hline
\end{tabular}

TABLE II: Theoretical periods and numerical values of $T_{p e r}$ and $T_{\text {damp }}$ for several donor densities.

The frequency of the oscillations of order $0.5 \times 10^{-12} s$ corresponding to wavelengths of order $10^{-4} \mathrm{~m}$ makes the electrostatic description for such an oscillating charge relevant at the nanometer scale. Note that the damping is increasing when the nonlinear effects are stronger, in agreement with what is observed in some other models studied for example in [20]) where the nonlinearity kills the beating effect after reaching a critical strength. Such a theoretical study would be valuable here but requires some heavy mathematical techniques.

\section{CONCLUSION}

The comparison of the reduced model introduced in [11] 12][13][15] with the simulation of the complete Schödinger-Poisson system confirms its ability to predict the bifurcation diagrams for far from equilibrium resonant tunneling devices. Such a discussion in [11] and [15] had already explained in the single well problem which barrier configuration could lead to hysteresis phenomena. Although a rather strong sensitivity to the geometry of the barriers, it also allows to design configurations for which resonant states of a double well system interact nonlinearly in a rather stable way. The question is whether such nonlinear steady states or in the transient regime such a nonlinear beating effect survive in a more complete modelling taking into account the nonlinear effects outside the barrier-well structure. In the time-dependent double well problem, the coupling of the oscillating dipole with the electromagnetic field could be of interest as well.

\section{Acknowledgments}

The authors would like to thank N. Ben Abdallah, F. Mehats, O. Pinaud, P. Racek, O. Vanbésien, B. Vinter and $\mathrm{U}$. Wulf for remarks and discussions about this work.
[1] R. Landauer, IBM J. Res. Develop. 1, 223 (1957).

[2] M. Büttiker, Y. Imry, R. Landauer, and S. Pinhas, Phys. Rev. B 31, 6207 (1985).

[3] F. Chevoir and B. Vinter, Phys. Rev. B 47, 7260 (1993).

[4] W. R. Frensley, Rev. Mod. Phys. 62, 745 (1990).

[5] N. Ben Abdallah, P. Degond, and P. A. Markowich, Z. Angew. Math. Phys. 48, 135 (1997).

[6] N. Ben Abdallah, J. Math. Phys. 41, 4241 (2000).

[7] F. Nier, Nonlinearity 11, 1127 (1998).

[8] N. B. Abdallah and O. Pinaud, J. Comput. Phys. 213, 288 (2006).

[9] S. E. Laux, A. Kumar, and M. V. Fischetti, J. Appl. Phys. 95, 5545 (2004).

[10] O. Pinaud, J. App. Phys. 92, 1987 (2002).

[11] F. Nier and M. Patel, in Multiscale methods in quantum mechanics (Birkhäuser Boston, Boston, MA, 2004), Trends Math., pp. 99-111.
[12] V. Bonnaillie-Noël, F. Nier, and M. Patel, To appear in Ann. Inst. H. Poincaré Anal. Non Linéaire (2008).

[13] V. Bonnaillie-Noël, F. Nier, and M. Patel, To appear in J. Math. Soc. Japan (2008).

[14] G. Jona-Lasinio, C. Presilla, and J. Sjöstrand, Ann. Physics 240, 1 (1995).

[15] V. Bonnaillie-Noël, F. Nier, and M. Patel, J. Comp. Phys. 219, 644 (2006).

[16] P. Mounaix, O. Vanbésien, and D. Lippens, Appl. Phys. Letter 57, 1517 (1990).

[17] H. Gummel, IEEE Trans. Electron Devices 11, 455 (1974).

[18] V. Grecchi, A. Martinez, and A. Sacchetti, Asymptotic Anal. 13, 373 (1996).

[19] A. Anton, VLSI Design 6, 313 (1998).

[20] V. Grecchi, A. Martinez, and A. Sacchetti, Comm. Math. Phys. 227, 191 (2002). 